\title{
Patient Perspective on the Burden of Skin and Joint Symptoms of Psoriatic Arthritis: Results of a Multi- National Patient Survey
}

\author{
Joseph F. Merola • David Shrom · Jennifer Eaton • Christine Dworkin • \\ Craig Krebsbach · Bijal Shah-Manek · Julie Birt
}

Received: October 9, 2018 / Published online: January 4, 2019

(C) The Author(s) 2019

\begin{abstract}
Introduction: Psoriatic arthritis (PsA) and psoriasis (PsO) have a significant impact on HRQOL and work productivity loss. In patients with both PsA and PsO, the full extent of the physical and emotional burden of joint- and skin-related symptoms is less understood from the patient perspective.

Methods: A cross-sectional study of $\mathrm{PsO}$ patients with PsA from the US, France, and Germany was conducted using an online survey. Data on demographics, PsO severity by patient-reported body surface area involvement
\end{abstract}

Enhanced digital features To view enhanced digital features for this article go to https://doi.org/10.6084/ m9.figshare.7430000.

Electronic supplementary material The online version of this article (https://doi.org/10.1007/s40744018-0135-1) contains supplementary material, which is available to authorized users.

J. F. Merola ( $\square)$

Brigham and Women's Hospital, Harvard Medical

School, Boston, MA, USA

e-mail: jfmerola@bwh.harvard.edu

D. Shrom $\cdot$ J. Eaton $\cdot$ J. Birt

Eli Lilly and Company, Indianapolis, IN, USA

C. Dworkin

Ipsos Healthcare, Indianapolis, IN, USA

C. Krebsbach · B. Shah-Manek

Ipsos Healthcare, New York City, NY, USA
(BSA), PsA severity by RAPID3, impact of PsO and PsA using Patient Global Assessment (1-5), and novel questions exploring the emotional burden of joint/skin-related symptoms were collected. Multivariate regression analyses examined severity of joint and skin symptoms as predictors of quality of life (QoL), measured by PsAQoL, and Work Productivity and Activity Impairment (WPAI).

Results: Of the 439 patients, $23.9 \%$ had mild (RAPID3 of $0-2$ ) and $76.1 \%$ had moderate-severe PsA (RAPID3 of 2.1-10), while 51\% had mild and $49 \%$ had moderate-severe $\mathrm{PsO}$ ( $\geq 3$ palms of the hand for BSA). Multivariate analyses showed that severity of joint symptoms was strongly associated with lower QoL $(t=13.15)$, followed by impact of skin symptoms $(t=5.11)$, and age $(t=-4.73)$, all $p<0.0001$. About $57 \%$ of all patients reported a DLQI $>5$, indicating a moderate-to-extremely large effect of psoriasis on HRQoL. Joint severity and impact of joint symptoms were the strongest predictors of WPAI. Patients also associated skin and/or joint symptoms with a variety of emotions and QoL measures that were not captured on the validated scales (fatigue, how they think of themselves, how others thought of them, making a first impression etc.).

Conclusions: In this study, both skin and joint symptoms had a broad, meaningful impact on patient QoL, work productivity, daily activities, and emotional well-being. These data 
highlighted the unique and significant impact of PsA among patients with PsO.

Funding: Eli Lilly and Company.

Keywords: Disease burden; Multinational study; Patient perspective; Psoriasis; Psoriatic arthritis

\section{INTRODUCTION}

Psoriatic arthritis (PsA) is a chronic, progressive, inflammatory arthritis that can co-occur as an associated condition in patients with psoriasis (PsO), and result in permanent joint disability and increased mortality risk $[1,2]$. A substantial number of patients with psoriasis will develop this form of arthritis, with estimates ranging from 6 to $41 \%[1,3]$. Among the general population, prevalence estimates of PsA can vary due to its highly heterogeneous disease course, diagnostic challenges, and the existence of several different definitions (diagnostic codes, classification criteria, etc.) that have been used to define the disease over time $[4,5]$. In the United States, prevalence estimates have ranged from 0.06 to $0.25 \%$, with a similar range of $0.05-0.21 \%$ reported in Europe.

PsA is a heterogeneous condition characterized by the clinical domains of arthritis, enthesitis, dactylitis spondylitis, and psoriasis [3]. For patients, pain, fatigue and progressive joint damage, can impair health-related quality of life (HRQoL) and physical functioning. The skin symptoms of psoriasis have a separate and significant impact on quality of life (QoL), which includes physical and emotional burden. Further, a relationship has been established between the extent of disability and impact on physical/mental QoL among this population with concurrent disease [6].

Even though there are some ongoing studies to identify risk factors [7], there exists a need for properly designed prospective studies to learn more about PsA development and progression [8], to decrease this important gap in the literature. For example, less is known as to whether the treatment setting and consequent care providers may play an important role in patient outcomes. The importance of early detection of
PsA in a dermatological setting has been well recognized, and guidelines have been drafted to help guide dermatologists in screening and providing appropriate referrals [9-12] and encourage collaboration among specialists [13]. Novel multidisciplinary clinical programs that include both dermatologists and rheumatologists have been developed in order to improve concurrent patient care, with encouraging preliminary results [14]. A recent study conducted by the Psoriasis and Psoriatic Arthritis Clinics Multicenter Advancement Network Consortium (PPACMAN) group showed that despite few challenges in dual care models, there were several benefits including improving patient care, opportunity to study long-term outcomes/ measures, collaborative care for complex diseases in addition to professional development and improved communications between the teams [14]. Challenges remain however, with evidence suggesting that PsA may be underdiagnosed among patients with $\mathrm{PsO}$ seen in a dermatology setting $[15,16]$.

The heterogeneous nature of PsA and its subsequent burden upon patients also presents a unique challenge to the development and implementation of standardized outcome assessments. The majority of empirical studies have therefore examined disease burden among PsO patients with PsA through chart review, registry or claims data, and thus may provide an incomplete picture of the psychosocial impact of these concurrent conditions. Among those with $\mathrm{PsO}$, mounting evidence suggests that concurrent PsA increases the risk of sexual dysfunction $[17,18]$, and psychiatric disorders such as anxiety and depression [19, 20]; patient reported outcomes that are rarely captured in medical charting or evaluated in routine practice. In addition, there may be specific aspects of disease impact that are unique to this patient population, and are not routinely evaluated by health care professionals or routine outcome measures. For example, many patients with PsO report avoiding physical touch due to their condition [21], yet few studies have examined the impact of such specific symptomatology on quality of life, relationships and intimacy among those with concurrent PsA. Thus, limited comprehensive data exists concerning the 
impact of PsA across emotional domains, relationships and daily activities. The objective of the current study was to examine the patients' perspective on the impact of both joint and skin symptoms on their physical function and emotional well-being in a real-world setting.

\section{METHODS}

\section{Study Design}

A multi-national cross-sectional survey was conducted in patients with both PsA and PsO from the United States, France, and Germany. Data from the United States were collected between April 2017 and May 2017 and data from European countries between May 2017 and June 2017. An initial qualitative study was conducted followed by a quantitative survey administration to examine the study objectives.

\section{Qualitative Phase}

To facilitate the design of a larger questionnaire, an initial qualitative study was conducted among 30 patients (from US, Germany, and France) to identify any under-represented concepts in existing instruments and better understand full extent of the impact of their PSA with respect to both joint-related and skin-related symptoms. During the interviews, patients provided their perspective on the ability of the Dermatology Life Quality Index (DLQI), the Short-Form-36 (SF-36) and the PsA Quality of Life Questionnaire (PsA QOL) to capture the impact of their disease, and they were asked to further elaborate on the impact on their disease which was not captured by these tools.

Ten participants from each respective country were recruited to have a total of 30 interviews across three countries. The interviews lasted approximately $45 \mathrm{~min}$ each and the US interviews were conducted via webcam and the non-US data were collected by a local moderator with simultaneous translation to English for study purposes; as use of webcams for the interview were not allowed due to respective country-level privacy laws.
Analysis of the patient interviews revealed that there is a diverse emotional impact of both skin- and joint-related manifestations of PsA and a diverse set of situations or daily-life activities that can be impacted by both skinand joint-related symptoms. To address this, a set of novel questions were developed to more comprehensively assess the emotional burden of PsA as well its impact on different aspects of daily life. These novel questions are described below in the description of the questionnaire elements.

\section{Quantitative Phase}

Following the qualitative study, a comprehensive survey was administered to the study eligible patients across all three countries. A select sample of patients were chosen for pre-testing the survey, to ensure content validity and gain understanding on survey experience prior to survey administration to the entire study sample.

Below is a description of the elements along with the novel questions that were included to capture emotional burden among the patients.

\section{Demographics and Medical History}

Information including age, gender, race, ethnicity, employment status, body mass index, smoking status, alcohol use was collected at the time of the survey as well as disease duration, current provider, provider who diagnosed the conditions, and comorbidities.

\section{PsO Severity}

Patients were asked to self-report psoriasis severity [referred as body surface area (BSA)], using a single question: 'How much Psoriasis do you currently have'. Response options included: None or very little psoriasis (Mild); Only a few patches that could be covered by one or two palms of your hand (Mild); Scattered patches that could be covered by between three and ten palms of your hand (Moderate); Extensive psoriasis covering large areas of the body that would be more than ten palms of your hand (Severe). 


\section{PSA Severity}

Patients reported the degree of severity of their joint symptoms of PsA using the Routine Assessment of Patient Index Data-3 (RAPID3), which consists of questions related to three main measures: physical function from Health Assessment Questionnaire (HAQ-DI), patient pain on a visual analogue scale (VAS) of 0-100 and patient global assessment on a VAS 0-100 [22]. Responses were scored and categorized as mild (RAPID3 of 0-2); moderate (RAPID3 of 2.1-4); Severe (RAPID3 of 4.1-10) [22].

\section{Patient Global Assessment (Specific for Joint or Skin Symptoms)}

Patients were asked to report two separate versions of the patient global assessments: one regarding the impact of their skin symptoms and the other regarding the impact of their joint symptoms. Global assessment of skin ("In all the ways your Psoriasis Symptoms as part of your PsA affect you, how would you rate the way you felt over the last week? Please select one") and joint symptoms ("In all the ways your Psoriatic Arthritis Pain affects you, how would you rate how you felt over the last week with respect to joint pain? Please select one") was assessed using five-point Likert responses (ranging from 1 (excellent) to 5 (poor)) asking to rate to what extent skin and joint symptoms affected them over the past week, respectively.

\section{Additional Measures: Novel Questions to Assess Impact on Daily Life and Emotional Status}

Additional questions were included to assess daily emotions due to the impact of PsO/skin symptoms and separately for PsA/joint symptoms. Participants were shown pictorial representation of 41 emotions (for e.g.: feeling happy, lonely/ignored, sad/depressed, hurt/ upset, irritated, confused, skeptical, surprised/ amazed, curious/interested, relieved/gratitude etc.) and asked to respond with top two feelings that described their skin and joint symptoms (Supplemental Fig. 1a and 1b). Questions on impact of skin and joint symptoms in the past 4 weeks on situations (spring, summer, fall, winter, depression, anxiety, holidays, genetics) and activities (personal hygiene, doing chores, friendships, family gatherings etc.) were also assessed on $0-10$ scale, with $0=$ no impact and $10=$ severe impact. Finally, overall health status ("How are you feeling overall today?") was also assessed on a $0-100$ scale, with $0=$ 'the worst I have felt in a long time' and 100=" the best I have felt in a long time'.

\section{Treatment Use}

Both current and past treatment use was collected, which included use of topical therapies, nonsteroidal anti-inflammatory drugs (NSAIDs), corticosteroids, oral small molecules, non-biologic and biologic systemic therapies.

\section{Quality of Life}

Two validated measures of quality of life namely, PsAQoL and DLQI was included. The PsAQoL is a 20-question assessment at the time of the survey, using only true/false questions [23]. The number of statements considered true by the respondent is their total PsAQoL score, where 0 is a good quality of life and 20 is poor, thus scores ranging from 0 to 20 . The DLQI is a validated ten-question assessment of the $\mathrm{PsO}$ effect on quality of life over the past week, with four response options for each question, scores ranging from 0 to $30[24,25]$. It is also assessed as a categorical variable: $0-1$ (none), 2-5 (small), 6-10 (moderate), 11-20 (very large), and 21-30 (extremely large) demonstrating the impact on quality of life.

\section{Work Productivity}

Work productivity impairment was assessed using the Work Productivity and Activity Impairment (WPAI: SHP) questionnaire [26], a validated six-item instrument which has 4 domains: absenteeism (i.e., percentage of work time missed because of health), presenteeism (i.e., percentage of impairment experienced while at work because of health), overall work productivity loss (i.e., an estimate that combines absenteeism and presenteeism), and impairment of non-work daily activities (i.e., percentage of impairment in daily activities because of health) over the course of the past week. 
The study was approved by Pearl Institutional Review Board (IRB) and met the standards of both FDA 21 CFR 56.104 and DHHS 45 CFR 46.101 regulations. Informed consent was obtained from all participants prior to participating in the study. This study was conducted in full conformance with the Guidelines for Good Pharmacoepidemiology Practices (GPP) published by the International Society of Pharmacoepidemiology (ISPE) and the laws and regulations of the country in which the research was conducted.

\section{Study Population}

Adult patients (aged $>18$ years but $<75$ years), who had a physician diagnosed PsA and PsO diagnosis, able to read/understand English (for the US), French (for France) and German (for Germany) and were willing to provide informed consent were recruited to participate in the study. Patients working for a pharmaceutical, health insurance, research, or advertising company were excluded to avoid self-presentation effects that could alter the responses. Additionally, medical professionals were also excluded as the objective of the study was to obtain purely a patient perspective.

A total of 439 study participants were recruited from various internet patient panels based on the study eligibility criteria. Panel participants are recruited by 'invitation only' or through online communities to be part of the panel, and not specifically through medical centers or physician referrals. The panels reach diverse audiences on a global scale making the sample representative within each country of interest. The interview request was sent via email to members of these panels who have previously identified as having PsA and PsO and expressed a willingness to participate in research studies. The interview request included a statement of informed consent and a set of screening questions (inclusion/exclusion criteria) to determine eligibility. Each participant provided informed consent and was compensated for the time spent on the completion of the survey.

\section{Statistical Analysis}

Descriptive analyses were conducted to describe the patient sample with respect to demographics, health history, disease history, and patient outcomes. Frequencies and percentages were reported for categorical outcomes and means, standard deviations, medians, and ranges were reported for continuous outcomes. Kruskal-Wallis or $\chi^{2}$ tests were used to compare group differences amongst continuous variables and categorical variables, respectively.

Correlations were assessed between independent and dependent variables to determine strong relationships and potential multicollinearities in a multivariate scope. Then a series of generalized linear regression (GLM) and logistic regression models were conducted to determine the relationship between individual symptoms and patient outcomes (quality of life, work productivity and activity impairment) adjusting for confounding variables. Analyses also investigated association between individual symptoms, as well as broader symptom categories such as skin versus joint, with QoL measures. Parameter estimates with standard errors and corresponding $t$ tests and $p$ values are reported. All analyses were conducted using SAS v9.3 or R v3.4.

\section{RESULTS}

A total of 439 patients diagnosed with both PsA and $\mathrm{PsO}$ completed the final survey: $n=300$ from the United States (68.3\%), $n=88$ from Germany $(20.1 \%)$, and $n=51$ from France (11.6\%). Overall, slightly more than half were females $(52.2 \%)$, with mean (SD) age of 49.37 (14.06) years, $83.1 \%$ were whites, and more than $50 \%$ of them were employed $(46.5 \%$ full time and $13.0 \%$ part-time) at the time of the survey. When presence of comorbidities was examined, about one-third of the patients had high cholesterol (33\%), 16\% type 2 diabetes and $10 \%$ heart disease. When current treatment was examined, about $50 \%$ of the patients were on biologic therapy and more than one-third on corticosteroids and prescription topical treatment (Table 1). Overall, 43.3\% were currently 
Table 1 Demographics, disease measures, PROs, and treatment use

\begin{tabular}{|c|c|}
\hline$N=439$ & \\
\hline Age, years, mean (SD) & $49.4(14.1)$ \\
\hline Male, $n(\%)$ & $210(47.8)$ \\
\hline BMI, $\mathrm{kg} / \mathrm{m}^{2}$, mean $(\mathrm{SD})$ & $29.8(13.9)$ \\
\hline \multicolumn{2}{|l|}{ Race, $n(\%)$} \\
\hline Caucasian & $365(83.1)$ \\
\hline African American & $12(2.7)$ \\
\hline Hispanic & $17(3.9)$ \\
\hline Asian & $10(2.3)$ \\
\hline Other & $27(6.2)$ \\
\hline None & $8(1.8)$ \\
\hline \multicolumn{2}{|l|}{ Comorbidities, $n$ (\%) } \\
\hline Hypertension & $97(22 \%)$ \\
\hline High cholesterol & $145(33 \%)$ \\
\hline Type 2 diabetes & $70(16 \%)$ \\
\hline Heart disease & $44(10 \%)$ \\
\hline Eye inflammation/uveitis & $31(7 \%)$ \\
\hline Heart attack/stroke & $31(7 \%)$ \\
\hline $\begin{array}{l}\text { Cancer (excluding NMSC and } \\
\text { melanoma) }\end{array}$ & $26(6 \%)$ \\
\hline NMSC & $26(6 \%)$ \\
\hline Melanoma & $18(4 \%)$ \\
\hline Hyperlipidemia & $22(5 \%)$ \\
\hline \multicolumn{2}{|l|}{ Skin severity } \\
\hline Mild, $n(\%)$ & $223(50.8)$ \\
\hline Moderate, $n(\%)$ & $146(33.3)$ \\
\hline Severe, $n(\%)$ & $70(15.9)$ \\
\hline \multicolumn{2}{|l|}{ Joint severity } \\
\hline Mild, $n$ (\%) & $105(23.9)$ \\
\hline Moderate, $n(\%)$ & $132(30.1)$ \\
\hline Severe, $n$ (\%) & $202(46)$ \\
\hline DLQI, mean (SD) & $9.7(8.4)$ \\
\hline PsA QoL, mean (SD) & $9.1(6.1)$ \\
\hline
\end{tabular}

Table 1 continued

\begin{tabular}{lc}
\hline$N=\mathbf{4 3 9}$ & \\
\hline WPAI, mean (SD) & $10.39(19.81)$ \\
Work absenteeism & $33.88(28.98)$ \\
Work presenteeism & $37.15(31.81)$ \\
Work productivity loss & $43.78(29.09)$ \\
Activity impairment & \\
Current treatment use ${ }^{\mathrm{a}}, n(\%)$ & $88(20 \%)$ \\
Light and laser therapy & $167(38 \%)$ \\
Corticosteroids & $145(33 \%)$ \\
Prescription topical therapy & $149(34 \%)$ \\
NSAIDs & $35(8 \%)$ \\
Oral small molecule $^{\text {Methotrexate }}$ & $79(18 \%)$ \\
Non-biologic systemic therapy $^{\mathrm{b}}$ & $70(16 \%)$ \\
Biologic therapy & $220(50 \%)$ \\
\hline
\end{tabular}

$B M I$ body mass index, NMSC non-melanoma skin cancer, $D L Q I$ dermatology life quality index, $P s A$ psoriatic arthritis, $Q o L$ quality of life, WPAI work productivity and activity impairment, NSAIDs non-steroidal anti-inflammatory drugs

${ }^{a}$ Patients could be on multiple therapies and hence total does not add up to $100 \%$

b Includes fumaric acid derivatives, cyclosporine, Soriatane and Neogason (acitretin), Azulfidine (sulfasalazine), and Amevive (alefacept)

c Includes Cimzia (certolizumab pegol), Cosentyx (secukinumab), Enbrel (etanercept), Humira (adalimumab), Remicade (infliximab), Simponi (golimumab), Stelara (ustekinumab), and Taltz (ixekizumab)

managed by rheumatologist only (51\% in the US, $29.4 \%$ in France and $25 \%$ in Germany), $13.9 \%$ by a dermatologist only (13\% in the US, $12 \%$ in France and $18.2 \%$ in Germany), 3.4\% by both rheumatologist and dermatologist $(2.7 \%$ in the US, $7.8 \%$ in France and 3.4\% in Germany) and $39.4 \%$ by other physician specialties (33\% in the US, $51 \%$ in France and $53.4 \%$ in Germany). Other physician specialties included general practice, internist, other only or a combination of specialties. 
The severity of skin symptoms, which was measured by the amount of affected body surface area, was mild for approximately half of the sample $(50.8 \%)$, followed by moderate $(33.3 \%)$ and severe $(15.9 \%)$. However, for joint severity, which was measured by RAPID3, 46\% reported being severe, $30.1 \%$ moderate, and $23.9 \%$ mild (Table 1). For skin symptoms, 38, 49, and $61 \%$ of mild, moderate, and severe patients reported fair/poor on the skin global assessment scale, respectively, demonstrating the increase in impact on overall health with increase in severity. Results were similar when joint severity was assessed using the patient global assessment tool, with 13,53 , and $81 \%$ of mild, moderate and severe patients reporting poor/fair on overall well-being in the past week (Fig. 1). As shown in the figure, higher proportion of patients with severe joint symptoms reported feeling poor (33\%) compared to severe skin symptoms (24\%). These differences in the distribution of responses were statistically significant.

Even though several factors were significant, after accounting for correlations and multicollinearity, age and gender were the strongest confounders which were adjusted in the multivariate modeling while examining the impact of skin/joint symptoms on QoL and work productivity.

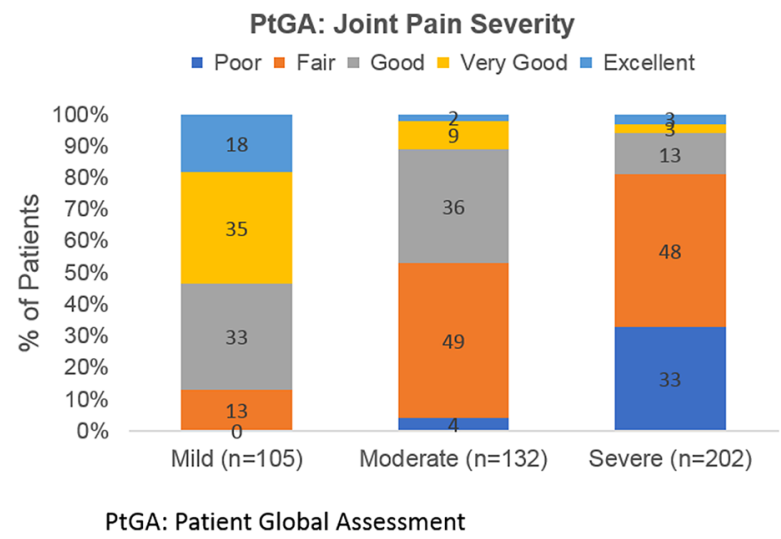

Fig. 1 Patient global assessment on joint and skin severity

\section{Impact of Joint and Skin Severity on QoL}

As expected, higher levels of reported skin and joint severity was associated with a greater impact on QoL as assessed by the DLQI and PsAQoL. As shown in Fig. 2, skin severity had higher impact on the skin-related QoL measure, compared to the joint symptoms even with milder disease (mean DLQI of 5.3 vs. 3.6 for mild skin symptoms vs. mild joint symptoms, respectively) and for moderate disease, this difference increased with moderate skin symptoms having higher impact on QoL as assessed by DLQI compared to moderate joint symptoms (mean DLQI of 12.5 vs. 7.4 for skin vs. joint symptoms, respectively). Of note, $57 \%$ of all patients reported a DLQI $>5$, indicating a moderate-to-extremely large effect of psoriasis on health-related QoL. In addition, among those reporting mild $\mathrm{PsO}, 31 \%$ reported DLQI $>5$, indicating a moderate to extremely large effect on QoL (Supplementary Fig. 2). The patterns were similar when QoL was assessed using PsAQoL, however the differences in mean PsAQoL scores were minimal for the severe skin vs. joint symptoms group.

In multivariate analyses, after controlling for age and gender, results were consistent and we found that the severity of joint and skin symptoms significantly contributed to the impact on PsAQoL (Table 2).

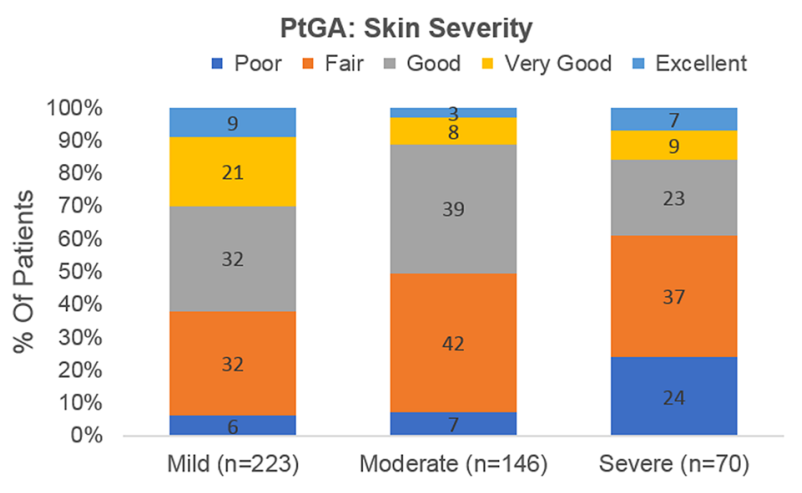


DLQI

PsA QoL

PsO Severity (BSA involvement)
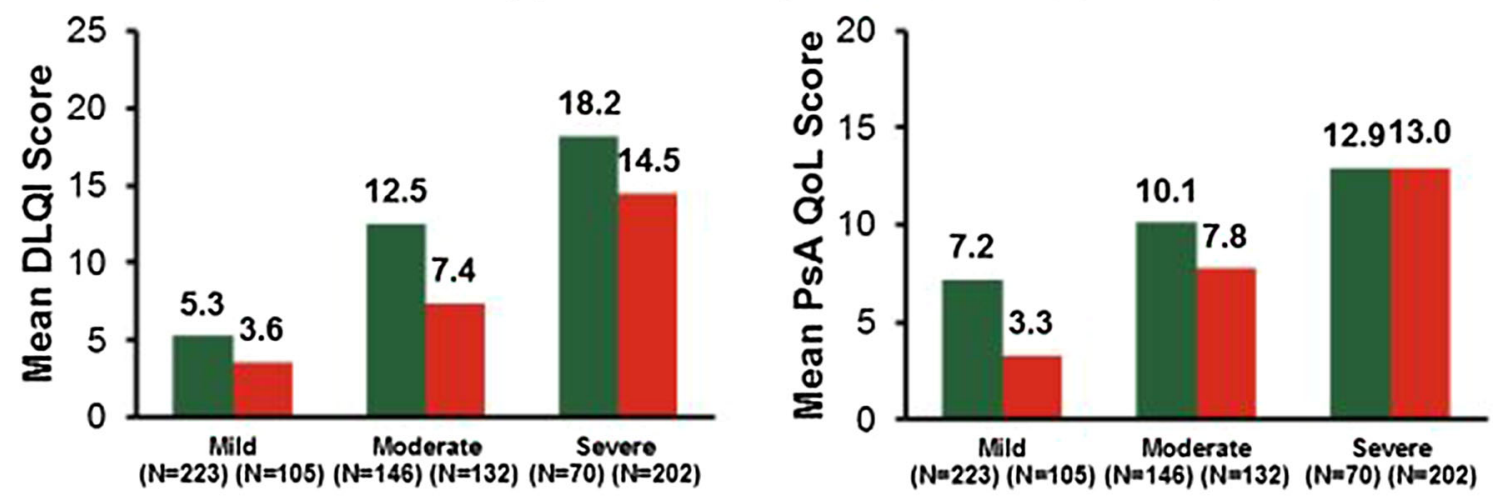

- Patient reported

$B S A=$ body surface area; $D L Q 1=$ Dermatology Life Quality Index; $P S A=$ psoriatic arthritis; $P S A$ QOL $=$ Psoriatic Arthritis Quality of Life questionnaire; $P S O=p$ soriasis; RAPID-3=Routine Assessment of Patient Index Data-3 score

Fig. 2 Quality of life by joint and skin symptom severity

Table 2 Association of skin/joint symptoms and quality of life measures (PsAQoL and WPAI)

\begin{tabular}{lclll}
\hline Coefficient & Estimate & SE & $t$ value & $p$ value \\
\hline PsAQoL $^{\text {a }}$ & & & & \\
Skin severity & 1.17 & 0.229 & 5.1092 & $<0.0001$ \\
Joint global & 1.5408 & 0.1171 & 13.1531 & $<0.0001$
\end{tabular}

WPAI-absenteeism $^{\mathrm{a}}$

$\begin{array}{crrlr}\text { Skin severity } & -0.1728 & 0.174 & \mathrm{n} / \mathrm{a} & 0.3206 \\ \text { Joint severity } & -0.2327 & 0.077 & \mathrm{n} / \mathrm{a} & 0.0025 \\ \text { WPAI-presenteeism } & & & & \\ \text { W } & 2.6732 & 1.7584 & 1.5202 & 0.1298 \\ \text { Skin severity } & 7.829 & 0.7697 & 10.1716 & <0.0001\end{array}$

WPAI-work productivity loss

\begin{tabular}{lrrrr} 
Skin severity & 2.9304 & 1.9102 & 1.5341 & 0.1263 \\
Joint severity & 8.4748 & 0.8361 & 10.1359 & $<0.0001$ \\
WPAI-overall activity impairment & & & \\
Joint severity & 8.7345 & 0.5423 & 16.1062 & $<0.0001$ \\
Joint global & 4.5832 & 1.001 & 4.5783 & $<0.0001$ \\
\hline
\end{tabular}

a Controlling for age and gender

b Controlling for age

\section{Impact on Work Productivity and Activity Impairment}

Among those who were employed, the mean percentage of work absenteeism and presenteeism was $10.39 \%(\mathrm{SD}=19.81 \%)$ and $33.88 \%$ $(\mathrm{SD}=28.98 \%)$, respectively. Mean overall percentage of productivity loss and activity impairment were $37.15 \%(\mathrm{SD}=31.81 \%)$ and $43.78 \% \quad(\mathrm{SD}=29.09 \%)$, respectively. With respect to WPAI, while there are differences in WPAI impact across mild, moderate, and severe skin symptoms and mild, moderate, and severe joint symptoms, the multivariate analyses (controlling for age and/or gender) showed that impact on WPAI was most significantly driven by joint symptoms. Worse joint severity scores resulted in increased absenteeism $(p=0.0025)$, presenteesim, activity impairment, and productivity loss (all $p<0.0001$ ) (Table 2 ).

\section{Impact of Skin and Joint Symptoms on Emotions}

Based on the analysis of patient interviews during the qualitative phase, it was revealed that validated patient reported questionnaires do not sufficiently explore the diversity of the emotional burden of PsA and its impact on 


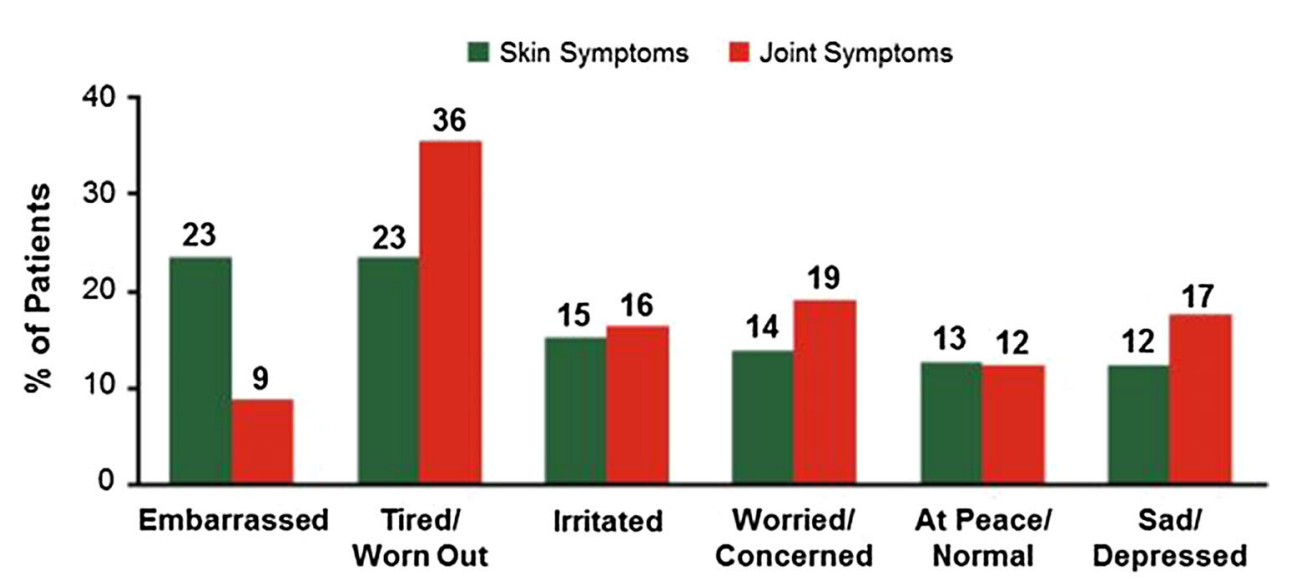

$P S A=p$ soriatic arthritis; $P S O=p$ soriasis

Fig. 3 Most often reported (> 10\%) feeling of emotions related to skin or joint symptom severity

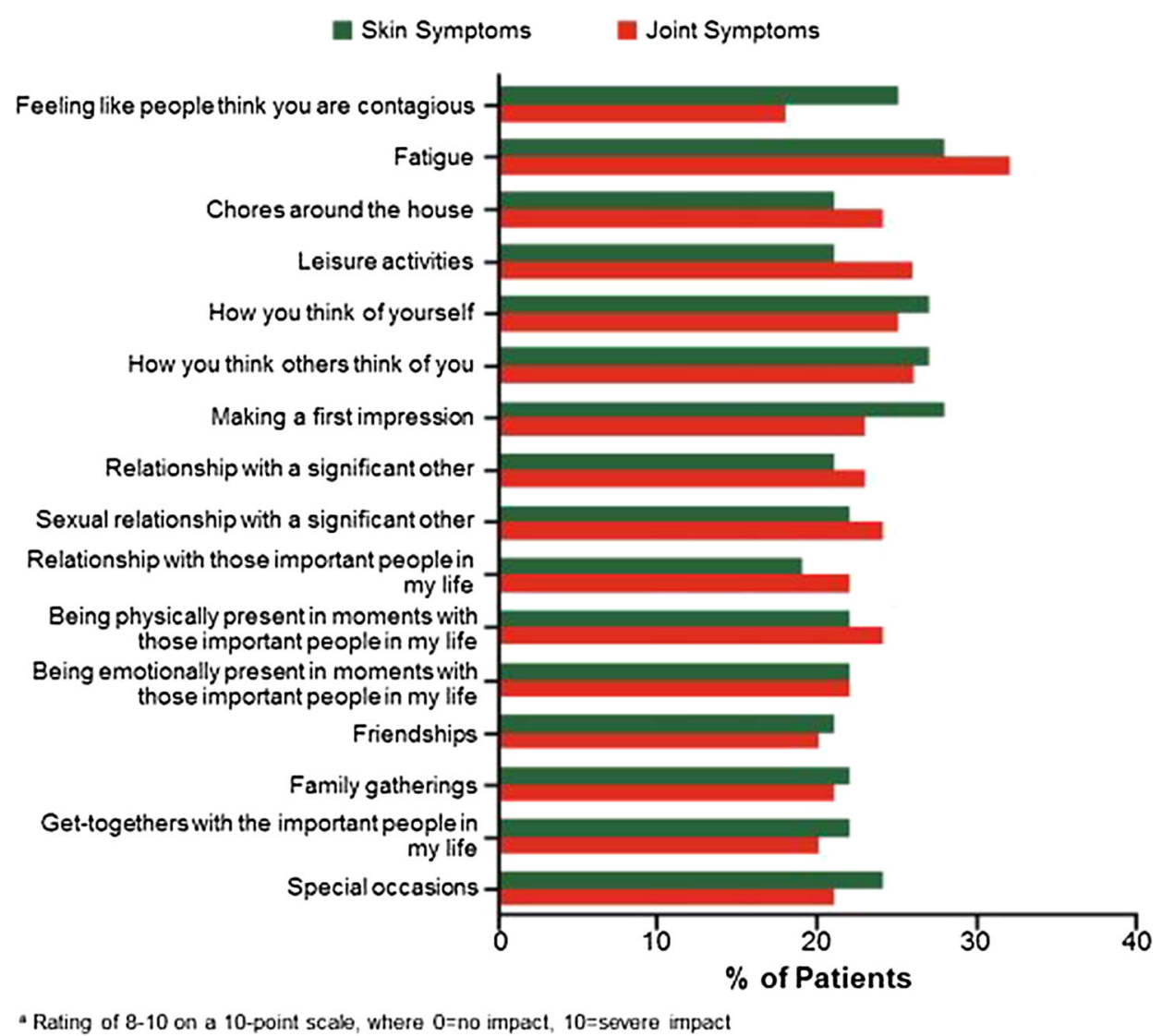

Fig. 4 Proportion of patients (> 20\%) reporting severe impact* on daily life situations based on skin and joint severity

aspects of daily life. In this study, emotional aspects were measured using novel questions using a ten-point Likert scale for both skin and joint symptoms and the impact they had on emotions, with 0 being no impact and 10 being severe impact. Compared to skin symptoms, joint symptoms had a significantly higher impact on feelings of tiredness and being worn out, as well as sadness, depression, shame, and guilt. Skin symptoms, on the other hand, were 
significantly more impactful than joint symptoms for feelings of shyness, embarrassment, shame, and guilt. Joint symptoms had a significantly higher impact on fatigue as well as the ability to do household chores and leisurely activities compared to skin symptoms. However, skin symptoms did have a significantly higher impact on feeling contagious compared to joint symptoms (Figs. 3,4 ). On several measures, there was no significant difference between skin and joint symptoms, suggesting that both affected emotional domains similarly, demonstrating that both symptoms had comparable impact on their emotions and were important to patients.

\section{DISCUSSION}

In the current study of patients with psoriatic arthritis, both skin and joint symptoms had a broad and meaningful impact across patient reported outcomes, including QoL, work productivity, daily activities, and emotional wellbeing. These results highlight the unique and significant impact of symptomatology among patients with concurrent PsA and PsO, and further emphasizes the need to adopt a holistic, personalized and comprehensive approach to patient care and treatment planning.

The current study demonstrated the relatively strong impact of both joint and skin symptomatology on QoL. Overall, both skin and joint symptoms had very strong associations with QoL. Age was also an important contributor, with younger patients reporting greater impairment. It may be possible that as people age and gain greater experience managing symptoms, they are able to more effectively limit their impact on domains of QoL. Gender differences emerged to a lesser degree, with females possessing worse QoL than males.

The inclusion of novel questions identified during the qualitative phase added additional insights into the individual impact of the disease. More recently developed questionnaires like Psoriatic Arthritis Impact of Disease Tool (PSAID) do capture much broader aspects of PsA experience among the patients like pain, dermatological issues, social activity, and relationships (spouse/partner) under physical and social sub-domains. However, more recently, Doogan and his colleagues [27], using the patient narratives from online communities, found that many of the subtle concepts within emotional, cognitive, and role activity were not captured in the disease specific or general health-related QoL instruments like PSAID, PsAQoL, and SF-36. Similarly, in our study, the emotional impact of both skin and joint symptoms was notable, with each symptom cluster possessing unique associations with psychosocial outcomes. Whereas several factors such as tiredness, fatigue, and chores are much more impacted by joint-related issues, skin symptoms can possess greater impact factors such as embarrassment and feeling contagious. This underscores that PsO skin symptoms, even though diverse can be just as important overall as corresponding joint symptoms in patients with PsA.

In general, worse symptoms and disease severity resulted in greater work activity impairment across all subscales, regardless of whether they were skin or joint issues. When we examined mild and moderate severities for skin and joint, the WPAI subscales were significantly lower for joint-related scores. While joint issues might seem more pressing on work-related topics, it appears for mild and moderate cases that skin is associated with worse WPAI subscales (data not shown). The broader trend was further confirmed in multivariate models, with analyses suggesting that worse joint and skin symptoms and severity were associated with worse WPAI scores, and increased age with better WPAI scores overall.

The importance of both joint severity and skin symptoms across a variety of patient reported outcomes emphasizes the need for a coordinated and holistic approach to treatment. Whereas dermatologists possess greater experience treating $\mathrm{PsO}$ and rheumatologists in treating PsA, both specialties must work collaboratively to effectively address the impact of joint and skin symptoms on a patient's life. As evidenced by the current study, the impact of symptoms can be far reaching, including impaired QoL, emotional symptoms, and work activity. 


\section{Limitations and Future Research}

Whereas the current study possesses many strengths, including its large-scale populationbased survey methodology, it is important to examine these results in the context of study limitations. Patients were recruited from an online panel, which may not be representative of the overall population of patients with PsA, and symptoms and severity levels were self-reported, which could lead to biased estimates. Further, reported symptoms and severity levels were not independently assessed by a health professional, nor confirmed through medical chart review.

This study does provide fertile ground for future work, including the potential utility of more quantitative based measures of skin severity. The four categorical based responses for skin severity based on the frequency of patches, while informative, lacks the quantitative power of the RAPID3 scores for joint severity. Moreover, the patient global focus on skin and joint symptoms were measured with two single questions, while a measure comprising multiple questions would add some reliability and the potential for better assessment of these latent attitudes. This could also increase the predictive power of the global focus on skin and joint symptoms.

\section{CONCLUSIONS}

In conclusion, concurrent diagnoses of PsA and $\mathrm{PsO}$ is associated with significant patient burden across multiple outcomes, including QoL, and work productivity. Patients also associated skin and/or joint symptoms with a variety of emotions and QoL measures that were not captured on the validated scales. QoL was impacted by both skin and joint symptoms, including among those patients with even mild symptoms. The effective management of skin and joint symptoms could enhance QoL, particularly among those younger in age, as well as improve work productivity. Overall, symptoms associated with PsO and PsA cause significant impairment among patients, and thus require the development and implementation of coordinated and holistic treatment approaches among respective health professionals.

\section{ACKNOWLEDGEMENTS}

We thank all the participants of this study.

Funding. Sponsorship for this study and article processing charges were funded by Eli Lilly and Company. All authors have reviewed and given their approval for this version to be published.

Medical Writing and/or Editorial Assistance. We would like to acknowledge Chitra Karki, Ipsos Healthcare, for her assistance in medical writing and submission process, which was sponsored by Eli Lilly and Company.

Authorship. All authors meet the International Committee of Medical Journal Editors (ICMJE) criteria for authorship of this article, had full access to all the data in this study and take complete responsibility for the integrity of the data and accuracy of the data analysis.

Disclosures. J F. Merola is a consultant and/ or investigator for Biogen IDEC, AbbVie, Amgen, Eli Lilly, Novartis, Pfizer, Janssen, UCB, Samumed, Celgene, Sanofi Regeneron, Merck and GSK. D. Shrom is a current employee and shareholder of Eli Lilly and Company. J. Eaton is a current employee and shareholder of Eli Lilly and Company. J. Birt is a current employee and shareholder of: Eli Lilly and Company. C Dworkin is an employee of Ipsos LLC. C. Krebsbach is an employee of Ipsos LLC. B. Shah-Manek was an employee of Ipsos LLC at the time of the study conduct. B. Shah-Manek is currently an employee of Noesis Healthcare Technologies, CA.

Compliance with ethics guidelines. The study was approved by Pearl Institutional Review Board (IRB) and met the standards of both FDA 21 CFR 56.104 and DHHS 45 CFR 46.101 regulations. Informed consent was obtained from all participants prior to participating in the study. This study was conducted 
in full conformance with the Guidelines for Good Pharmacoepidemiology Practices (GPP) published by the International Society of Pharmacoepidemiology (ISPE) and the laws and regulations of the country in which the research was conducted.

Data availability. All data generated or analyzed during this study are included in this published article/as supplementary information files.

Open Access. This article is distributed under the terms of the Creative Commons Attribution-NonCommercial 4.0 International License (http://creativecommons.org/licenses/ by-nc/4.0/), which permits any noncommercial use, distribution, and reproduction in any medium, provided you give appropriate credit to the original author(s) and the source, provide a link to the Creative Commons license, and indicate if changes were made.

\section{REFERENCES}

1. Ogdie A, Weiss P. The epidemiology of psoriatic arthritis. Rheum Dis Clin North Am. 2015;41:545-68.

2. Liu JT, Yeh HM, Liu SY, Chen KT. Psoriatic arthritis: epidemiology, diagnosis, and treatment. World J Orthop. 2014;5:537-43.

3. Mease PJ, Armstrong AW. Managing patients with psoriatic disease: the diagnosis and pharmacologic treatment of psoriatic arthritis in patients with psoriasis. Drugs. 2014;74:423-41.

4. Boehncke WH. Psoriasis and psoriatic arthritis: flip sides of the coin? Acta Derm Venereol. 2016;96:436-41.

5. Napolitano M, Caso F, Scarpa R, Megna M, Patri A, Balato N, et al. Psoriatic arthritis and psoriasis: differential diagnosis. Clin Rheumatol. 2016;35:1893-901.

6. Edson-Heredia E, Zhu B, Guo J, Maeda-Chubachi T, Lebwohl M. Disease burden and quality of life in psoriasis patients with and without comorbid psoriatic arthritis: results from National Psoriasis Foundation panel surveys. Cutis. 2015;95:173-8.
7. Azevedo VF, Buiar PG. Risk factors and predictors of psoriatic arthritis in patients with psoriasis. An Bras Dermatol. 2013;88:233-6.

8. Langham S, Langham J, Goertz HP, Ratcliffe M. Large-scale, prospective, observational studies in patients with psoriasis and psoriatic arthritis: a systematic and critical review. BMC Med Res Methodol. 2011;11:32.

9. Gisondi P, Altomare G, Ayala F, Conti A, Dapavo P, De Simone $C$, et al. Consensus on the management of patients with psoriatic arthritis in a dermatology setting. J Eur Acad Dermatol Venereol. 2017;32: 515-528. https://doi.org/10.1111/jdv.14741.

10. Garg A, Gladman D. Recognizing psoriatic arthritis in the dermatology clinic. J Am Acad Dermatol. 2010;63:733-48.

11. Landells I, MacCallum C, Khraishi M. The role of the dermatologist in identification and treatment of the early stages of psoriatic arthritis. Skin Therapy Lett. 2008;13:4-7.

12. Laws P, Barton A, Warren RB. Psoriatic arthritiswhat the dermatologist needs to know. J Eur Acad Dermatol Venereol. 2010;24:1270-7.

13. Migliore A, Cusano F, Bianchi G, Malara G, Epis O, De Pita O. Management of psoriatic arthritis: should the interaction between dermatologists and rheumatologists in clinical practice be intensified? J Biol Regul Homeost Agents. 2015;29:547-61.

14. Okhovat JP, Ogdie A, Reddy SM, Rosen CF, Scher JU, Merola JF. Psoriasis and psoriatic arthritis clinics multicenter advancement network consortium (PPACMAN) survey: benefits and challenges of combined rheumatology-dermatology clinics. J Rheumatol. 2017;44:693-4.

15. Mease PJ, Gladman DD, Papp KA, Khraishi MM, Thaci D, Behrens F, et al. Prevalence of rheumatologist-diagnosed psoriatic arthritis in patients with psoriasis in European/North American dermatology clinics. J Am Acad Dermatol. 2013;69:729-35.

16. Khraishi M, Chouela E, Bejar M, Landells I, Hewhook T, Rampakakis E, et al. High prevalence of psoriatic arthritis in a cohort of patients with psoriasis seen in a dermatology practice. J Cutan Med Surg. 2012;16:122-7.

17. Kurizky PS, Mota LM. Sexual dysfunction in patients with psoriasis and psoriatic arthritis-a systematic review. Rev Bras Reumatol. 2012;52:943-8.

18. Molina-Leyva A, Jimenez-Moleon JJ, Naranjo-Sintes $\mathrm{R}$, Ruiz-Carrascosa JC. Sexual dysfunction in psoriasis: a systematic review. J Eur Acad Dermatol Venereol. 2015;29:649-55. 
19. Lamb RC, Matcham F, Turner MA, Rayner L, Simpson A, Hotopf M, et al. Screening for anxiety and depression in people with psoriasis: a crosssectional study in a tertiary referral setting. Br J Dermatol. 2017;176:1028-34.

20. McDonough E, Ayearst R, Eder L, Chandran V, Rosen CF, Thavaneswaran A, et al. Depression and anxiety in psoriatic disease: prevalence and associated factors. J Rheumatol. 2014;41:887-96.

21. Armstrong AW, Cather JC, Paul C, Edson-Heredia E, Zhu B, Hollister K, et al. Association of touch avoidance with disease severity and quality of life in psoriasis patients. J Psoriasis Psoriatic Arthritis. 2017;2:57-63.

22. Pincus T, Swearingen CJ, Bergman M, Yazici Y. RAPID3 (Routine Assessment of Patient Index Data 3), a rheumatoid arthritis index without formal joint counts for routine care: proposed severity categories compared to disease activity score and clinical disease activity index categories. J Rheumatol. $2008 ; 35: 2136-47$.

23. McKenna SP, Doward LC, Whalley D, Tennant A, Emery P, Veale DJ. Development of the PsAQoL: a quality of life instrument specific to psoriatic arthritis. Ann Rheum Dis. 2004;63:162-9.
24. Finlay AY, Khan GK. Dermatology Life Quality Index (DLQI) - a simple practical measure for routine clinical use. Clin Exp Dermatol. 1994;19:210-6.

25. Hongbo Y, Thomas CL, Harrison MA, Salek MS, Finlay AY. Translating the science of quality of life into practice: what do dermatology life quality index scores mean? J Invest Dermatol. 2005;125:659-64.

26. Reilly MC, Zbrozek AS, Dukes EM. The validity and reproducibility of a work productivity and activity impairment instrument. Pharmacoeconomics. 1993;4:353-65.

27. Doogan S, Heid J, Benosman S, Ogdie A, Martin L, Sunkureddi $\mathrm{P}$, et al. Using self-reported patient experiences to evaluate patient reported outcomes (PRO) instruments: learnings from digital patient communities in psoriatic arthritis [abstract]. Arthritis Rheumatol. 2016;68 (suppl 10). https:// acrabstracts.org/abstract/using-self-reported-patientexperiences-to-evaluate-patient-reported-outcomespro-instruments-learnings-from-digital-patient-com munities-in-psoriatic-arthritis/. Accessed 20 July 2018. 